\title{
A NEW RANDOM GRAPH MODEL WITH SELF-OPTIMIZING NODES: CONNECTIVITY AND DIAMETER
}

\author{
Richard J. La and Maya Kabkab \\ Department of Electrical \& Computer Engineering (ECE) and the Institute \\ for Systems Research (ISR), University of Maryland, College Park, Maryland
}

\begin{abstract}
We introduce a new random graph model. In our model, $n, n \geq 2$, vertices choose a subset of potential edges by considering the (estimated) benefits or utilities of the edges. More precisely, each vertex selects $k, k \geq 1$, incident edges it wishes to set up, and an undirected edge between two vertices is present in the graph if and only if both of the end vertices choose the edge. First, we examine the scaling law of the smallest $k$ needed for graph connectivity with increasing $n$ and prove that it is $\Theta(\log (n))$. Second, we study the diameter of the random graph and demonstrate that, under certain conditions on $k$, the diameter is close to $\log (n) / \log (\log (n))$ with high probability. In addition, as a byproduct of our findings, we show that, for all sufficiently large $n$, if $k>\beta^{\star} \log (n)$, where $\beta^{\star} \approx 2.4626$, there exists a connected Erdös-Rényi random graph that is embedded in our random graph, with high probability.
\end{abstract}

\section{INTRODUCTION}

In recent years, there has been a renewed interest in various random graph models, some of which is driven by research activities on network formation or generation, e.g., markets and social networks $[18,38]$. Some of these random graph models include the famous Erdös-Rényi random graphs [10], random intersection graphs [8], random graphs with hidden variables $[9,12]$, random threshold graphs [35], and random geometric graphs $[3,39]$, just to name a few.

These random graphs are constructed in very different manners. Each model has a unique mechanism by which edges are selected in the graph. Also, the edge selection mechanisms often make use of attributes or variables assigned to each vertex and lead to different correlations in edges and distributions of random graphs. Short descriptions of these random graph models and some of their applications are provided in Section 3.

Although in some cases associating attributes with vertices makes sense, there are other cases for which it is more natural to associate attributes with the edges. For example, in a (multihop) wireless network, the importance of an edge or link (in the network) might be dependent on how much traffic is carried using the link. Consequently, even the importance of two links incident on the same node can vary drastically, depending on how traffic is

Address correspondence to Richard La, Department of Electrical \& Computer Engineering (ECE) and the Institute for Systems Research (ISR), University of Maryland, College Park, Maryland 20742, USA. E-mail: hyongla@umd.edu

Color versions of one or more of the figures in the article can be found online at www.tandfonline.com/uinm. 
routed in the network. In such cases, the importance of an edge is not determined by the end vertices. Instead, it is shaped by how the edge is used or what the edge is used for, and the importance of a vertex is, in turn, set by the importance of edges incident on the vertex.

Our new random graph model aims to take a step toward modeling such situations and assigns attributes to (potential) edges between vertices (as opposed to the vertices themselves). Moreover, it captures, to some extent, the strategic or self-optimizing nature of vertices that we expect in many applications; we interpret the attributes of the edges as their benefits or utilities, and each vertex tries to select a subset of incident edges with the largest benefits.

Such situations could arise naturally; for instance, in (cooperative) distributed systems supported by wireless networks (e.g., coordinated motion control of autonomous mobile agents), in which individual agents attempt to take actions they believe to be most beneficial based on locally available information [4]. This requires that each agent identify a subset of other agents with which it wishes to coordinate actions or exchange information in order to optimize the overall system performance. Here, the edges may represent physical/wireless links or indicate operational relations.

Unfortunately, in many cases, setting up edges and/or exchanging information is not free and incurs nonzero communication and computational costs, e.g., energy consumption and overhead. In these cases, the agents ought to select only important edges and should not choose their neighbors at random, especially when communication resources are constrained [28,29]. To this end, the agents should first gauge the benefit of each potential edge, which may be estimated based on previous interactions with other agents or available prior information, and pick the edges with the highest benefits.

In addition, in some cases, the number of edges agents can select or wish to set up might be limited for several possible reasons. As an example, if maintaining edges requires exchanging control information with a large number of neighbors, the resulting overhead could be prohibitive. Moreover, high degrees of nodes in a large dense network can also complicate the operation of network protocols and necessitate a considerable amount of state information at the nodes, with only marginal gains in performance. For instance, proactive or table-driven routing protocols for multihop wireless networks strive to maintain up-to-date topological information of the network [13,40]. Because even a small change in network topology triggers flooding of an update message, the overhead for keeping up-to-date topological information increases rapidly with node degrees in a large network. Also, the computation of optimal routes will become more challenging as the number of available paths grows. Thus, in some cases it might be desirable to limit the degrees of nodes so long as the performance does not degrade significantly in doing so.

We consider simple cases in this study, in which edges are selected only once by the vertices. However, in some applications, edge selections may be repeated over time and the edge set is updated dynamically based on current information. This can happen, for example, when the benefits or utilities of the edges are time varying (e.g., multihop wireless networks) or while the vertices are in the process of discovering and learning the benefits of the edges.

Another line of research related to network formation can be found in the literature on game-theoretic or strategic network formation (e.g., [25,31] and references therein). Most of these studies model the problem of network formation as a game among the vertices, in which each vertex is a rational player and is interested in maximizing its payoff, e.g., the number of reachable vertices discounted according to their hop distances. 
These game theoretic models typically assume that the vertices have both the necessary knowledge and computational capability to compute their payoffs as a function of strategy profile, i.e., the set of actions chosen by the vertices. This payoff computation often requires global knowledge, which might not be available to the vertices in many applications. Moreover, due to the stringent assumptions on the availability of global information and full rationality, the networks predicted at equilibria are often very simple (e.g., complete topology, star topology, or disconnected network, comprising very small components with one or two vertices). The final equilibrium network is typically shaped by the costs of establishing edges between vertices.

In contrast, our model allows us to capture the strategic nature of the vertices to a certain extent without assuming their full rationality or the availability of global information at their disposal. This is because the vertices are assumed to base their selections only on the estimated benefits of potential edges, which do not depend on other edges selected by vertices, and choose the edges that they believe to be most helpful.

Suppose that there are $n, n \geq 2$, vertices in the graph and each vertex is allowed to choose $k, 1 \leq k \leq n-1$, potential edges that provide the largest estimated benefits. In our model, however, setting up an edge requires mutual consent between its two end vertices; when only one of the end vertices chooses the edge, the edge is not set up. The questions we are interested in exploring are the following:

Q1: How large should $k$ be as a function of $n$ in order for the graph to be connected (with high probability)?

Q2: When the graph is connected, what is its diameter?

To offer a partial answer to these questions, we prove the following main results: for large $n$,

F1. if $k>\beta^{\star} \log (n)$, where $\beta^{\star} \approx 2.4626$, the graph is connected with high probability (whp);

F2. if $k<0.5 \log (n)$, the graph contains at least one isolated node (i.e., a node with no neighbor), and is not connected whp; and

$\mathrm{F} 3$. when $k$ is of the order $\log (n)$ and is larger than $\beta^{\star} \log (n)$ (so that the graph is connected whp), the diameter of the graph is close to $\log (n) / \log (\log (n))$.

In the process of proving the first result, we also bring to light the following interesting fact: under the condition $k>\beta^{\star} \log (n)$, in spite of their seemingly disparate constructions, we can find an Erdös-Rényi random graph that is embedded in our random graph and is also connected whp. This suggests a somewhat surprising connection between the Erdös-Rényi random graph model and our random graph model.

The rest of this article is organized as follows: Section 2 describes the random graph model we propose. We briefly discuss several existing random graph models and point out key differences between them and our model in Section 3. Section 4 defines the graph connectivity and the diameter of a graph and formally states the questions of interest to us. We present the main results in Section 5, followed by numerical results in Section 6 . We compare our findings on the proposed graph model against the properties of existing random graph models in Section 7. Throughout the article, we assume that all random variables (rvs) are defined on a common probability space $(\Omega, \mathcal{F}, \mathbb{P})$. 


\section{A NEW RANDOM GRAPH MODEL}

For each $n \in \mathbb{N}_{+}:=\{2,3, \ldots\}$, let $\mathcal{V}^{(n)}=\{1,2, \ldots, n\}$ be the set of $n$ nodes or vertices. ${ }^{1}$ We assume that all edges between nodes are undirected in our model and denote the undirected edge between nodes $i$ and $j(i \neq j)$ by $(i, j)=(j, i)$. For fixed $n \in \mathbb{N}_{+}$, a subset of $n(n-1) / 2$ possible undirected edges is chosen through the following edge selection process.

1. Edge values - For every pair of distinct nodes, say $i, j \in \mathcal{V}^{(n)}(i \neq j)$, there is a value $V_{i, j}$ associated with the edge between them. This edge value $V_{i, j}$ is used to model the benefit that node $i$ anticipates from having an edge with node $j$ or the overall importance of the edge to the network itself or a system running on the network.

We assume that edge values are symmetric, i.e., $V_{i, j}=V_{j, i} \cdot{ }^{2}$ Although this may not be true in some cases, we believe that this a reasonable assumption in many applications, including cooperative distributed systems; in such systems, the value of an edge should signify to both of its end vertices its utility to the overall system performance.

The values of $n(n-1) / 2$ possible edges are modeled as $n(n-1) / 2$ independent and identically distributed (i.i.d.) rvs with a common continuous distribution $F$. Although this is clearly a simplification of a complicated reality, we make this assumption to facilitate our analysis as a first step. Moreover, as it will be made clear, even under this simplifying assumption, the analysis is still nontrivial, in part because of complex correlations in edge selections, and we leave the relaxation of this i.i.d assumption as future work.

2. Edge selection - Based on realized edge values $\left\{V_{i, j} \mid i, j \in \mathcal{V}^{(n)}, i \neq j\right\}$, each node $i \in \mathcal{V}^{(n)}$ selects $k$ incident edges with the $k$ largest values. Denote the set of edges chosen by node $i$ by $E_{i}^{(n)} \subset\left\{(i, j) \mid j \in \mathcal{V}^{(n)} \backslash\{i\}\right\}$. The edge set $\mathcal{E}^{(n)}$ of the graph is then given by

$$
\mathcal{E}^{(n)}:=\left\{(i, j) \mid(i, j) \in E_{i}^{(n)} \cap E_{j}^{(n)}\right\} \subseteq \mathcal{V}^{(n)} \times \mathcal{V}^{(n)} .
$$

It is clear from the definition in (2.1) that an edge is in $\mathcal{E}^{(n)}$ if and only if both of its end nodes pick the edge. In other words, we consider only those scenarios where mutual consent of the end nodes is needed for an edge to be present in the graph.

When $(i, j) \in \mathcal{E}^{(n)}$, we say that nodes $i$ and $j$ are neighbors and denote this relation by $i \leftrightarrow j$. Thus, when node $i$ chooses an edge $(i, j) \in E_{i}^{(n)}$, it expresses its desire to be node $j$ 's neighbor, and we say that node $i$ picks node $j$ (as a potential neighbor).

The pair $\left(\mathcal{V}^{(n)}, \mathcal{E}^{(n)}\right)$ gives rise to a random graph because the edge set $\mathcal{E}^{(n)}$ is a random set. We denote the random graph $\left(\mathcal{V}^{(n)}, \mathcal{E}^{(n)}\right)$ by $\mathbb{G}(n, k)$. Let us first point out a few observations regarding $\mathbb{G}(n, k)$ :

\footnotetext{
${ }^{1}$ Throughout the rest of the article, we use the words nodes and vertices interchangeably.

${ }^{2}$ In more general cases, however, edges may be directed, and edge values $V_{i, j}$ and $V_{j, i}$ may be correlated, but not necessarily identical.
} 
O-1. Beacuse each node is allowed to choose only $k$ potential neighbors, a node degree is upper bounded by $k$. The exact node degree distribution is, however, difficult to compute.

O-2. As the selection of potential neighbors is carried out using symmetric edge values, there are correlations in the selection of $E_{i}^{(n)}, i \in \mathcal{V}^{(n)}$. For instance, when $k<n-1$, given $\left\{(i, j) \in E_{i}^{(n)}\right\}$, the conditional probability of $\left\{(i, j) \in E_{j}^{(n)}\right\}$ is larger than the prior probability, i.e.,

$$
\mathbb{P}\left[(i, j) \in E_{j}^{(n)} \mid(i, j) \in E_{i}^{(n)}\right]>\mathbb{P}\left[(i, j) \in E_{j}^{(n)}\right] .
$$

The reason for this inequality is that, conditioned on the event $\left\{(i, j) \in E_{i}^{(n)}\right\}$, the conditional distribution of $V_{i, j}$ first-order stochastically dominates the prior distribution of $V_{i, j}$ [41]. In other words, $\mathbb{P}\left[V_{i, j} \geq v \mid(i, j) \in E_{i}^{(n)}\right] \geq \mathbb{P}\left[V_{i, j} \geq v\right]$ for all $v \in \mathbf{R}$, and the inequality is strict for some values of $v \in \mathbf{R}$. This is a consequence of the assumption that nodes choose edges with the largest edge values, and $V_{i, j}$ is one of the $k$ largest values among $n-1$ edge values seen by node $i$.

O-3. The choice of edge value distribution $F$ is not important in that the distribution of $\mathbb{G}(n, k)$ and our findings do not depend on the distribution $F$. This is because only the ordering of edge values matters as each node selects $k$ edges with the $k$ largest edge values among $n-1$ possible incident edges. In order to see this, note that, given any two continuous distributions $F_{1}$ and $F_{2}$, we can construct a set of i.i.d. rvs with distribution $F_{1}$, using another set of i.i.d. rvs with distribution $F_{2}$ in such a way that the ordering of the rvs is preserved with probability one (w.p.1). This can be done, for instance, with help of the probability integral transform and quantile functions [2]. The preservation of the ordering among the rvs in turn implies that, for any fixed ordering of the rvs, the probability that we will observe the given ordering of the rvs is the same for both $F_{1}$ and $F_{2}$. As a result, the distribution of $\mathbb{G}(n, k)$ is not dependent on the edge value distribution $F$ as long as it is continuous. We will make use of this observation in the proofs of our main results.

\section{RELATED EXISTING RANDOM GRAPH MODELS}

In this section we briefly summarize some well-known random graph models, and highlight the differences between these models and our model delineated in Section 2.

1. Erdös-Rényi random graphs: One of earliest random graph models is the Erdös-Rényi random graph model. In the so-called $G(n, p)$ model, which is also known as the Gilbert model [24], there are $n$ vertices in the graph, and each undirected edge between two vertices $i$ and $j$ is present in the edge set $\mathcal{E}$ with probability $p$, independently of other edges [10]. Because each undirected edge is added with probability $p$ independently, a node degree has a binomial $(n-1, p)$ distribution. Thus, not only is the degree distribution in the Erdös-Rényi random graphs quite different from that of our random graphs, but also the Erdös-Rényi random graphs do not model any correlations among the edges.

Another related Erdös-Rényi random graph model is known as $G(n, m)$ random graph model. In this model, there are $n$ vertices as before. However, instead of adding each undirected edge with probability $p$, a set of $m$ edges is selected at random. More precisely, each subset of $m$ edges is equally likely to be selected from the set containing 
$\left(\begin{array}{c}n(n-1) / 2 \\ m\end{array}\right)$ subsets of $m$ edges out of $n(n-1) / 2$ possible edges. However, it is shown that when $m \approx n(n-1) \cdot p / 2, G(n, p)$ and $G(n, m)$ behave similarly.

2. Random $m$-orientable graphs: A random $m$-orientable graph, also called a random $k$-out graph, is obtained by allowing each node to choose a set of $m$ neighbors with equal probability of $1 /\left(\begin{array}{c}n-1 \\ m\end{array}\right)$, independently of each other [23]. A pair of nodes $i$ and $j$ have an undirected edge between them if either node chooses the other node as a neighbor.

Even though each node chooses a fixed number of (potential) neighbors in both a random $m$-orientable graph and our graph, there are two key differences. First, a random $m$-orientable graph requires only one of the end nodes to choose an edge in order for the edge to be present in the graph. In contrast, in our graph, it is necessary for both end nodes to choose the edge. Second, the selections of neighbors are mutually independent in a random $m$-orientable graph, whereas they are correlated through edge values in our graph.

3. Random intersection graphs: In the simplest random intersection graph model, denoted by $G(n, m, k)$, there are $n$ vertices and each vertex is assigned $k$ distinct colors at random from a set of $m$ colors [8]. The set of $k$ colors assigned to each vertex is selected independently of those of other vertices. There exists an undirected edge between two vertices $i$ and $j$ if the two vertices share at least one common color.

The construction of a random intersection graph introduces correlations in the selection of edges as does our model. However, the degree of a node is still binomial $(n-1, p)$ distributed, where $p=1-\prod_{\ell=0}^{k-1}(1-k /(m-\ell))$.

Random intersection graphs have been used to model the Eschenauer-Gligor key distribution in (wireless) networks [21], e.g., [43]. Each node in a network is assigned $k$ keys out of a pool of $m$ keys, and two nodes can communicate with each other only if they share a common key. Hence, the connectivity of the network can be studied by examining the graph connectivity of $G(n, m, k)$ random intersection graph.

4. Random graphs with hidden variables: In a random graph with hidden variables studied in [12] (and later generalized in [9]), there are $n$ vertices, each of which is assigned a "fitness" (or "vertex-importance") denoted by $\varsigma_{i}$. These fitness values are modeled using $n$ i.i.d. rvs with some common distribution. For given fitness values of the vertices, there is an undirected edge between two vertices $i$ and $j$ with probability $f\left(\varsigma_{i}, \varsigma_{j}\right)$ for some function $f$. It is clear that this construction of a random graph introduces correlations in the edges via the fitness values of vertices.

This type of random graph is used to generate graphs with widely varying degree distributions, including power law distribution (so-called scale-free networks) [12], without having to resort to preferential attachment $[1,38]$. In fact, somewhat surprisingly, it is shown [12] that scale-free networks can be generated even by nonscale-free distributions for fitness values.

5. Random threshold graphs: A random threshold graph is a special case of random graphs with hidden variables [35]. In a random threshold graph, the edge selection function $f$ takes the form

$$
f\left(\varsigma_{i}, \varsigma_{j}\right)= \begin{cases}1 & \text { if } \varsigma_{i}+\varsigma_{j}>\Delta, \\ 0 & \text { if } \varsigma_{i}+\varsigma_{j} \leq \Delta,\end{cases}
$$

where $\Delta$ is some threshold. 
6. Random geometric graphs: In a random geometric graph with $n$ vertices on a spatial domain $\mathcal{D}$, which is often a subset of $\mathbf{R}^{k}$ for some $k \in \mathbb{N}:=\{1,2, \ldots\}$, the locations of the $n$ vertices are given by $n$ i.i.d. $\operatorname{rvs} \mathbf{X}_{i}^{(n)}, i=1,2, \ldots, n$, that are distributed over $\mathcal{D}$ according to a common spatial distribution. ${ }^{3}$ Two vertices $i$ and $j$ then have an undirected edge between them if

$$
\left\|\mathbf{X}_{i}^{(n)}-\mathbf{X}_{j}^{(n)}\right\| \leq \gamma
$$

where $\|\cdot\|$ denotes some norm (e.g., $L^{2}$ norm) used to measure the distance between two vertices, and $\gamma$ is some threshold.

Random geometric graphs are often used to model the one-hop connectivity in wireless networks, where the threshold $\gamma$ can be viewed as a proxy to the communication range employed by wireless nodes; e.g., [27,33,34].

7. $k$-nearest neighbor random graphs: Another important random graph model, which is related to both the proposed model and random geometric graphs, is the $k$-nearest neighbor graph $(k N N G)$ model $[5,6,7,42]$. The $k N N G$ are used to model the one-hop connectivity in wireless networks, in which each node initiates two-way communication with $k$ nearest nodes; e.g., [42].

Loosely speaking, there are two types of $k \mathrm{NNGs}$ - directed and undirected. Similarly as in a random geometric graph, a $k N N G$ with $n$ vertices is defined using $n$ i.i.d. rvs $\mathbf{X}_{i}^{(n)}, i=1,2, \ldots, n$. These rvs represent the locations of $n$ vertices and are distributed over $\mathcal{D} \subseteq \mathbf{R}^{d}$, where $d$ is typically not larger than three. For each vertex $i$, let $N_{i}^{(n)}(k)$ be the set of $k$ vertices that are closest to vertex $i$ according to some fixed norm. In a directed $k \mathrm{NNG}$, there is a directed edge from vertex $i$ to each vertex in $N_{i}^{(n)}(k)$. For an undirected $k \mathrm{NNG}$, we replace the directed edges with undirected edges.

It is clear that both random geometric graphs and $k$ NNGs introduce correlations in the existing edges via the locations of the vertices, i.e., $\mathbf{X}_{i}^{(n)}, i=1,2, \ldots, n$.

8. Configuration model: Given a set of $n$ vertices and their degree sequence $\mathbf{d}=\left(d_{i} ; i=\right.$ $1,2, \ldots, n)$, the configuration model produces a random graph with specified degrees [32,36,37]: initially, each vertex $i$ is assigned $d_{i}$ "stubs" or "half edges." Then, the edge set is constructed as a uniformly random matching on the stubs, i.e., each matching is selected with equal probability. Note that the resulting graph is not necessarily simple and may contain multiedges or self-loops.

The configuration model is often used to generate scale-free random networks with a power law degree distribution or other random networks with the same degree sequence as a real-world network.

It is noteworthy that the last six random graph models introduce correlations in edge selection through attributes associated with vertices. In contrast, in our random graph model, correlations are introduced through attributes associated with edges.

\footnotetext{
${ }^{3}$ The independence or homogeneity of node locations fails to hold in many practical scenarios. Heterogeneous mobility cases, still under an independence assumption were studied in [33]. In another work, the independence assumption in one-dimensional situations was relaxed and correlations were introduced via group mobility [34].
} 


\section{GRAPH CONNECTIVITY AND DIAMETER}

Before we state the questions of interest, we first introduce the definition of graph connectivity we adopt throughout the paper and define the diameter of an undirected graph.

Definition 4.1 We say that an undirected graph $G=(\mathcal{V}, \mathcal{E})$ is connected if it is possible to reach any node from any other node through a sequence of neighbors. In other words, for every pair of distinct nodes $i$ and $j$, we can find $K \in \mathbb{N}$ and a sequence of nodes $i_{1}, i_{2}, \ldots, i_{K}$ such that

i. $i_{1}=i$ and $i_{K}=j$, and

ii. $\left(i_{k}, i_{k+1}\right) \in \mathcal{E}$ for all $k=1,2, \ldots, K-1$.

The subgraph $\left(\mathcal{V}_{p}, \mathcal{E}_{p}\right)=: \mathcal{P}$ in Definition 4.1 , where $\mathcal{V}_{p}=\left\{i_{1}, i_{2}, \ldots, i_{K}\right\}$ and $\mathcal{E}_{p}=\left\{\left(i_{1}, i_{2}\right),\left(i_{2}, i_{3}\right), \ldots,\left(i_{K-1}, i_{K}\right)\right\}$, is called a path between nodes $i$ and $j$. The length of path $\mathcal{P}$ is defined as $K-1$.

Suppose $G=(\mathcal{V}, \mathcal{E})$ is an undirected graph. The distance between two distinct nodes $i, j \in \mathcal{V}$ is the minimum among the lengths of all paths between the two nodes, i.e., the length of the shortest path between nodes $i$ and $j$. We denote this distance by $d(i, j)$. When there is no path between two nodes $i$ and $j$, we set $d(i, j)=\infty$.

Definition 4.2 The diameter of an undirected graph $G$ is defined to be

$$
D(G)=\max \{d(i, j) \mid i, j \in \mathcal{V}, i \neq j\} .
$$

In other words, the diameter of $G$ is the largest distance among all pairs of nodes.

We are interested in the following questions: Let $\mathbb{G}(n, k)$ be a random graph described in Section 2.

Question \#1: How large should $k$ be in order to ensure connectivity of $\mathbb{G}(n, k)$ whp for large values of $n$ ?

Question \#2: When $\mathbb{G}(n, k)$ is connected, what is its diameter?

Unfortunately, computing the exact probability that the random graph $\mathbb{G}(n, k)$ is connected for fixed values of $n$ and $k$ is difficult. Similarly, finding the diameter of $\mathbb{G}(n, k)$, although in principle computationally feasible once the edge set is revealed, can be computationally demanding for large $n$.

For these reasons, we turn to the asymptotic analysis with an increasing number of nodes $n$. We first examine how $k$ should scale as a function of $n$ (denoted by $k(n)$ ) in order for the random graph $\mathbb{G}(n, k(n))$ to be connected whp. In particular, we are interested in finding the smallest $k(n)$ that ensures connectivity of $\mathbb{G}(n, k(n))$ whp. Second, we investigate the diameter of $\mathbb{G}(n, k(n))$ when $k(n)$ is of the same order as the smallest value that yields a connected graph.

\section{MAIN RESULTS ON CONNECTIVITY AND DIAMETER}

In this section, we provide a partial answer to the questions we posed in the previous section. More specifically, we show that, whp, (i) the smallest $k(n)$ required for graph connectivity is of the order $\log (n)$, and (ii) the diameter of $\mathbb{G}(n, k(n))$ is approximately 
$\log (n) / \log (\log (n))$ when $k(n)$ is not much larger than the smallest value that produces graph connectivity.

\subsection{Connectivity of $\mathbb{G}(n, k(n))$}

First, we derive a sufficient condition on $k(n)$ so that $\mathbb{G}(n, k(n))$ is connected (with probability close to one) for large values of $n$. Let $\beta^{\star}$ be the unique solution to $\beta=$ $\exp (\sqrt{2 / \beta})$, which is approximately 2.4626 .

Theorem 5.1. Suppose that $\beta>\beta^{\star}$ and $k(n) \geq \beta \cdot \log (n)$ for all sufficiently large $n$. Then,

$$
\mathbb{P}[\mathbb{G}(n, k(n)) \text { is connected }] \rightarrow 1 \text { as } n \rightarrow \infty .
$$

Proof. A proof of the theorem is provided in Appendix A.

The proof of Theorem 5.1 reveals the following very interesting fact. Under the condition in Theorem 5.1, with probability approaching one as $n \rightarrow \infty$, we can find an Erdös-Rényi random graph $G(n, p(n))$ with $p(n)=\zeta \log (n) / n, \zeta>1$, whose edge set is contained in the edge set of $\mathbb{G}(n, k(n))$. Since the Erdös-Rényi random graph $G(n, p(n))$ is connected with probability going to one when $\zeta>1$ [10], this implies that our random graph is connected with probability tending to one as $n$ increases.

Theorem 5.1 tells us that the smallest $k(n)$ necessary for graph connectivity only needs to scale as $O(\log (n))$. The following theorem states that it is $\Omega(\log (n))$ as well.

Theorem 5.2. Suppose that $\alpha<0.5$ and $k(n) \leq \alpha \cdot \log (n)$ for all sufficiently large $n$. Then,

$$
\mathbb{P}[\mathbb{G}(n, k(n)) \text { contains an isolated node }] \rightarrow 1 \text { as } n \rightarrow \infty .
$$

Proof. A proof of the theorem is given in Appendix C.

Because the probability that a graph is not connected is lower bounded by the probability that there is at least one isolated node in the graph, Theorem 5.2 implies that, under the stated condition, $\mathbb{G}(n, k(n))$ is connected with probability going to 0 as $n \rightarrow \infty$.

Our findings in Theorems 5.1 and 5.2 indicate that, for large $n$, the smallest $k(n)$ we need in order for $\mathbb{G}(n, k(n))$ to be connected lies in $\left[0.5 \log (n), \beta^{\star} \log (n)\right]$ whp. Hence, the smallest $k(n)$ required for graph connectivity scales as $\Theta(\log (n))$.

\subsection{Diameter of $\mathbb{G}(n, k(n))$}

In this subsection, we study the diameter of $\mathbb{G}(n, k(n))$ when the condition in Theorem 5.1 holds. 
Theorem 5.3. Suppose that $k(n)=\Theta(\log (n))$ such that $\liminf _{n \rightarrow \infty} k(n) / \log (n)>\beta^{\star}$. Then, the diameters of $\mathbb{G}(n, k(n)), n \in \mathbb{N}_{+}$, satisfy the following: for any positive $\epsilon>0$,

$$
\lim _{n \rightarrow \infty} \mathbb{P}\left[\left|\frac{D(\mathbb{G}(n, k(n)))}{\log (n) / \log (\log (n))}-1\right|>\epsilon\right]=0 .
$$

In other words, $D(\mathbb{G}(n, k(n))) /(\log (n) / \log (\log (n)))$ converges to one in probability.

Proof. Please see Appendix F for a proof.

Theorem 5.3 implies that if $k(n) \approx \kappa \log (n)$ for some $\kappa>\beta^{\star}$ for all sufficiently large $n$, the diameter of $\mathbb{G}(n, k(n))$ is close to $\log (n) / \log (\log (n))$ with high probability. For example, when $n$ is $10^{6}$ and $10^{9}$, we have $\log (n) / \log (\log (n)) \approx 5.2615$ and 6.8365 , respectively. Hence, the diameter of $\mathbb{G}(n, k(n))$ increases rather slowly with the number of nodes in the graph under the condition in Theorem 5.3. This is commonly called the small world phenomenon in the literature [18].

\section{NUMERICAL RESULTS}

\subsection{Connectivity}

Our findings in Section 5.1 suggest an interval to which the smallest $k(n)$ that yields graph connectivity belongs (whp). However, it does not establish the existence of a sharp phase transition that we observe, for instance, with the Erdös-Rényi random graphs [10]. In this section, we provide some numerical results to look for an answer to this question. In our numerical examples, for $n=50,200,800,3000$, and 8000 , we vary the value of parameter $k$ to see how the probability of graph connectivity changes with $k$. The empirical probability is obtained as the fraction of times the graph is connected out of 200 realizations for each point. This is plotted in Figure 1. The dotted vertical red lines in Figure 1(a) indicate $k=\log (n)$ for different values of $n$.

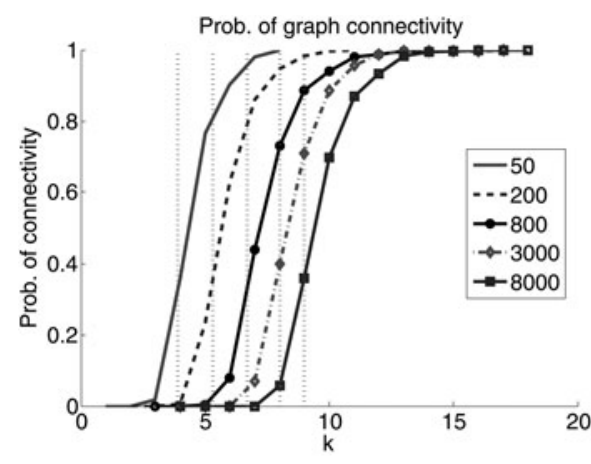

(a)

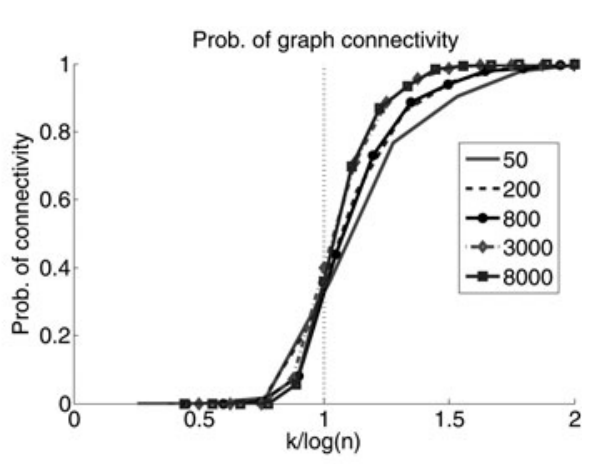

(b)

Figure 1 Probability of graph connectivity as a function of (a) $k$ and (b) $k / \log (n)$ for $n=50,200,800,3000$, and 8000 . 
There are two observations we can make from Figure 1. First, the probability of graph connectivity rises sharply as $k$ increases over a short interval around $\log (n)$. For instance, for $n=3000$, we have $0.5 \log (n)=4.00$ and $\beta^{\star} \log (n)=19.72$. It is clear from Figure 1(a) that, already for $n=3000$, the rapid ascent in the probability of graph connectivity with increasing $k$ takes place over an interval much shorter than the interval $\left[0.5 \log (n), \beta^{\star} \log (n)\right]=[4.00,19.72]$. This is corroborated by Figure $1(\mathrm{~b})$, which suggests that (most of) the transition occurs over $[0.8 \log (n), 1.2 \log (n)]$.

Second, the plots of the graph connectivity probability for different values of $n$ shown in Figure 1(a) resemble horizontally shifted versions of each other. Hence, the transition width, i.e., the length of the interval over which the probability increases from (close to) zero to (close to) one, does not appear to depend on $n$ and may behave like $\Theta(1)$ for large $n$. This implies that the transition width normalized by $\log (n)$ diminishes to zero with increasing $n$.

We illustrate this point using Figure 1(b). Note that the plots of connectivity probability with varying $n$ intersect where $k / \log (n)$ is approximately one. In addition, as $n$ increases, the slope of the transition around the intersection point climbs as well, thereby exhibiting decreasing transition width when normalized by $\log (n)$.

These two observations-(i) the sharp transition in the probability of graph connectivity occurs over a short interval centered around $\log (n)$, and (ii) the transition width may behave like $\Theta(1)$ - together suggest that, for large $n$, the distribution of the smallest $k(n)$ needed for graph connectivity could be concentrated around $\log (n)$. This leads to the following conjecture.

Conjecture 6.1 Suppose that $\beta>1$ and $k(n) \geq \beta \cdot \log (n)$ for all sufficiently large $n$. Then, $\mathbb{P}[\mathbb{G}(n, k(n))$ is connected $] \rightarrow 1$ as $n \rightarrow \infty$. Similarly, if $\beta<1$ and $k(n) \leq \beta \cdot \log (n)$ for all sufficiently large $n, \mathbb{P}[\mathbb{G}(n, k(n))$ is connected $] \rightarrow 0$ as $n \rightarrow \infty$.

\subsection{Node Isolation}

It is well known that for the Erdös-Rényi random graphs, when the graph does not have an isolated node, the graph is likely connected as well. This is captured by the following sharp result:

1. If the edge selection probability $p(n) \leq \alpha \log (n) / n$ with $\alpha<1, \mathbb{P}[G(n, p(n))$ contains an isolated node $] \rightarrow 1$ as $n \rightarrow \infty$.

2. If the edge selection probability $p(n) \geq \beta \log (n) / n$ with $\beta>1$, $\mathbb{P}[G(n, p(n))$ is connected $] \rightarrow 1$ as $n \rightarrow \infty$.

A similar observation is true for random geometric graphs and many other graphs. Hence, it is of interest to see if a similar observation holds with our random graphs.

Figure 2 plots the probability that there is no isolated node in $\mathbb{G}(n, k(n))$ for the same set of values of $n$ and $k$ used in Figure 1. By comparing Figures 1(a) and 2, it is clear that the probability that $\mathbb{G}(n, k(n))$ contains no isolated node is very close to the probability that it is connected. Hence, the figures suggest that, for large $n$, whp the minimum $k$ necessary for graph connectivity is close, if not equal, to the smallest $k$ required to eliminate isolated nodes in the graph. We formalize this observation in the form of following conjecture. 


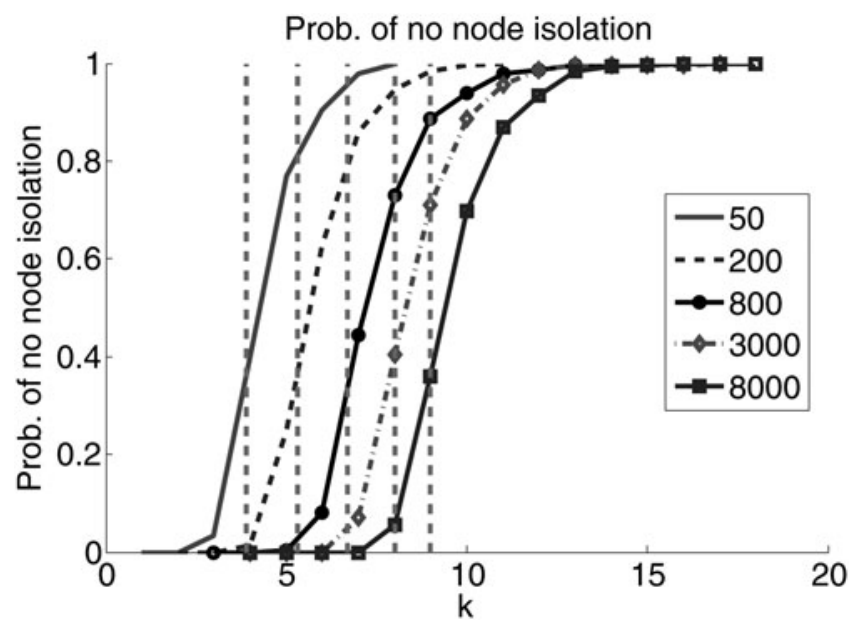

Figure 2 Probability of no isolated node as a function of $k$ for $n=50,200,800,3000$, and 8000 .

Conjecture 6.2 Suppose that $k^{C}(n)$ and $k^{N I}(n)$ denote the minimum $k$ required for graph connectivity and no node isolation, respectively. Then, $k^{N I}(n) / k^{C}(n)$ converges to one in probability.

\subsection{Diameter}

Figure 3 shows the probability mass function (PMF) of the graph diameter $D(\mathbb{G}(n, k))$ for $n=50$ and 1000. For $n=50$ and 1000 , we have $\beta^{\star} \log (n)=9.634$ and 17.011, respectively. In order to ensure that the graphs are connected whp, we set $k=11$ and 18 for $n=50$ and 1000, respectively. With these values of $k$, all 400 realized graphs (200 realizations for each value of $n$ ) are connected. The plots in Figure 3 are the empirical PMFs obtained from the 200 realizations for each plot.

For $n=50$ and 1000 , we get $\log (n) / \log (\log (n))=2.8679$ and 3.5742, respectively. Hence, Theorem 5.3 suggests that, assuming $n$ is sufficiently large, the distribution of the graph diameter should be concentrated around $\lceil\log (n) / \log (\log (n))\rceil$, which is 3 and 4 for $n=50$ and $n=1000$, respectively. Indeed, the PMFs in Figure 3 indicate that, although our finding is asymptotic in nature, even for $n=50$ the diameter of $\mathbb{G}(n, k)$ is concentrated on 3; 196 realized graphs have a diameter of 3, and the remaining four realized graphs have a diameter of 4 . For $n=1000,198$ realized graphs have a diameter of 4 with the other two realized graphs having a diameter of 5 .

\subsection{Average Node Degree and Node Degree Distribution}

In this subsection, we discuss (i) average node degrees and (ii) node degree distributions in the proposed graph model. Figure 4 plots the average node degree for $n=200$ and 8000 as the parameter $k$ is varied. It is clear from the figure that the average node degree can be well approximated using an affine function of $k$. The best linear regression line for $n=200$ (resp. $n=8000$ ) we obtain, using the polyfit function in MATLAB, is 


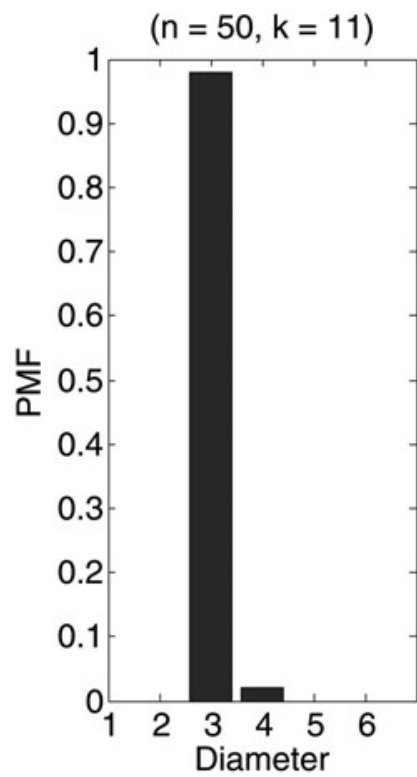

(a)

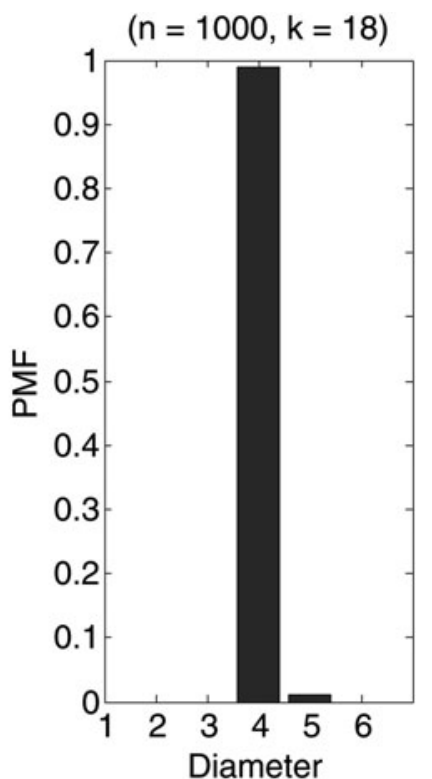

(b)

Figure 3 Probability mass function of graph diameter; (a) $n=50$, and (b) $n=1000$ (empirical PMF of 200 realizations).

average_degree $(k)=0.8918 \cdot k-0.6236($ resp. average_degree $(k)=0.9168 \cdot k-0.8412)$. The linear regression line for $n=8000$ is shown in Figure 4.

We plot the node degree distribution for different values of $k$ for $n=200$ and 8000 in Figure 5. The three values of $k$ used to generate the plots correspond to $\lfloor 0.5 \cdot \log (n)\rfloor$, $\lceil\log (n)\rceil$, and $\lceil 2 \cdot \log (n)\rceil$ for both values of $n$. First, the plots indicate that the PMF of node degree is increasing in degree between zero and $k$ in all six cases we consider. Second, they reveal that the node degree distribution in the proposed graph model is quite different from that of the Erdös-Rényi graph model, as we explain in the subsequent section. Third, for $k=\lfloor 0.5 \cdot \log (n)\rfloor$, the fraction of isolated nodes, i.e., nodes with zero degree, is nonnegligible, as expected from Figure 2.

\section{DISCUSSION}

In this section, we briefly compare our findings on the proposed random graph model to the properties of some of the random graph models outlined in Section 3.

\subsection{Graph Connectivity}

It is well known that, for sufficiently large $n$, both Erdös-Rényi random graphs and random geometric graphs (with uniform spatial distribution) are connected (resp. disconnected) whp if the average node degree is suitably larger (resp. smaller) than $\log (n)[10,39]$. Similarly, the smallest $k$ needed for connectivity of $k$ NNGs is shown to be $\Theta(\log (n))$ for both 


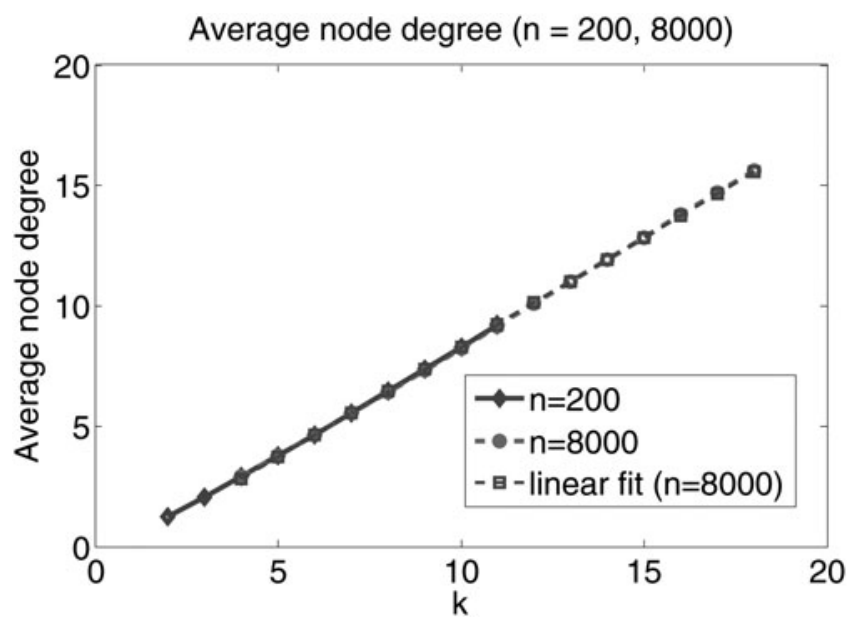

Figure 4 Average node degree as a function of $k$ for $n=200$ and $n=8000$.

directed and undirected graphs [5,6,7]. For example, if $k(n) \leq c_{l} \cdot \log (n)$ with $c_{l}<0.3043$ (resp. $k(n) \geq c_{u} \cdot \log (n)$ with $c_{u}>0.5139$ ), an undirected $k N N G$ is disconnected (resp. connected) whp for sufficiently large $n$ [6]. Similar bounds are derived for (strong) connectivity of directed $k N N G s$ in [6]. Moreover, the existence of critical constant $c^{\star} \in[0.3043,0.5139]$ is established for undirected $k N N G$ in [7].

Another interesting random graph model related to $k$ NNGs and our proposed model is the mutual $k$-nearest neighbor graphs, whose connectivity is studied in [11]. In a mutual $k N N G$, an undirected edge exists between two distinct vertices $i$ and $j$ if and only if $i \in N_{j}^{(n)}(k)$ and $j \in N_{i}^{(n)}(k)$ in the directed $k$ NNG. In other words, mutual selection is required, as in our proposed model. The smallest $k$ necessary for graph connectivity of a mutual $k \mathrm{NNG}$, denoted by $k^{\star}(n)$, is shown to be $O(\log (n))$ [11]. Note that $k^{\star}(n)$ cannot be smaller than the smallest $k$ required for connectivity of the undirected $k \mathrm{NNG}$. Because the lower bound for the smallest necessary $k$ in undirected $k N N G s$ is at least $0.3043 \cdot \log (n)$ (whp) [6], together with the finding in [11], we have $k^{\star}(n)=\Theta(\log (n))$.

Theorems 5.1 and 5.2 reveal that the smallest $k$ necessary for graph connectivity in the proposed model is also $\Theta(\log (n))$. However, as shown in Figure 4, the average degree of nodes around the conjectured threshold, i.e., $\log (n)$, appears to be different from those of the Erdös-Rényi graphs, random geometric graphs, and $k$ NNGs.

\subsection{Node Degree Distribution}

Recall from Section 3 that the node degree distribution in the $G(n, p)$ model is $\operatorname{binomial}(n-1, p)$. Similarly, when the spatial distribution of nodes in a random geometric graph is uniform over the spatial domain $\mathcal{D}$, except for the nodes close to the boundary of $\mathcal{D}$, a node degree is $\operatorname{binomial}(n-1, p)$ distributed, where $p$ is equal to the communication area of a node, i.e., the area within $\gamma$ from the node, divided by the area of $\mathcal{D}$. For large $n$ and small $p$, this binomial distribution can be approximated using a Poisson distribution with parameter $\lambda=n \cdot p$. We plot this Poisson distribution for two cases: (a) $n=200$ and $k=11=\lceil 2 \cdot \log (200)\rceil$ and (b) $n=8000$ and $k=18=\lceil 2 \cdot \log (8000)\rceil$. For these two 


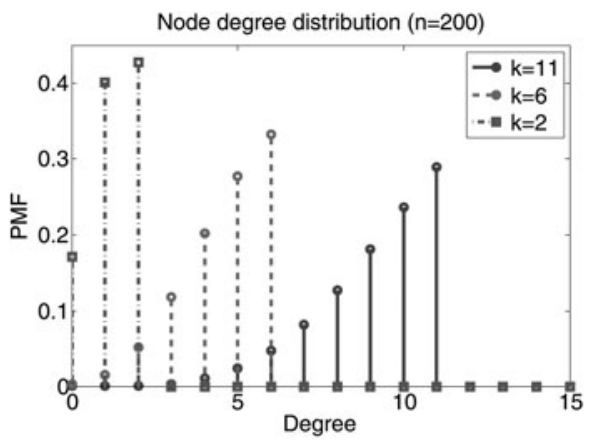

(a)

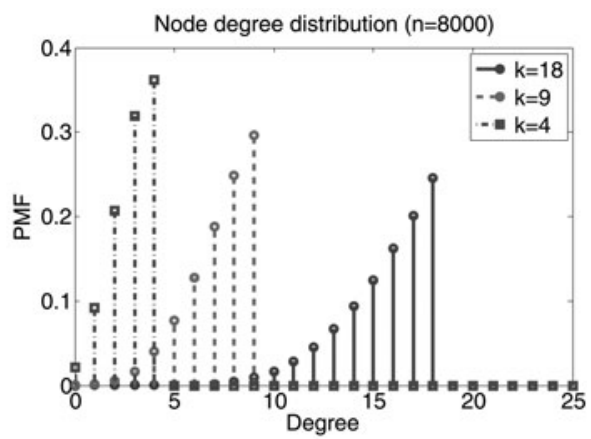

(b)

Figure 5 Node degree distribution for (a) $n=200$ and (b) $n=8000$.

cases, the average node degrees from Figure 4 are 9.19 and 15.57, respectively. We set the value of Poisson parameter $\lambda$ to these values and compare the Poisson distribution to the empirical node degree distribution shown in Figure 5. This is displayed in Figure 6.

It is evident from the figure that the node degree distributions behave quite differently in these random graph models when the average node degrees are similar. For instance, although a node degree cannot exceed $k$ in the proposed graph model, there is nonnegligible probability that a node degree is significantly larger than the average node degree in the $G(n, p)$ model and the random geometric graph model.

However the proof of Theorem 5.1 in Appendix A suggests that, for all sufficiently large $n$, if $k>\beta^{\star} \cdot \log (n)$, where $\beta^{\star} \approx 2.4626$, we can find an Erdös-Rényi graph $G(n, p)$ with $p=(1+\epsilon) \log (n) / n$ and $\epsilon>0$, which is embedded in $\mathbb{G}(n, k)$ and is connected whp. Obviously, when such an embedded Erdös-Rényi graph exists, a node degree in the ErdösRényi graph cannot exceed $k$. This can be seen in Figure 6 . When the parameter of the Poisson distribution is set to $\lambda=\log (n)$, which is 5.3 and 8.99 for $n=200$ and $n=8000$, respectively, the probability that the Poisson rv exceeds $k=\lceil 2 \cdot \log (n)\rceil<\beta^{\star} \cdot \log (n)$ is

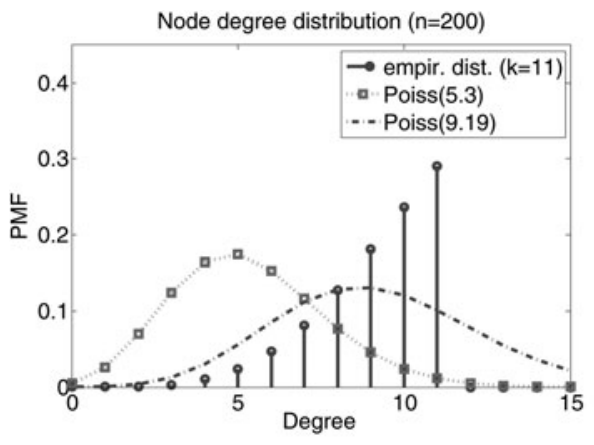

(a)

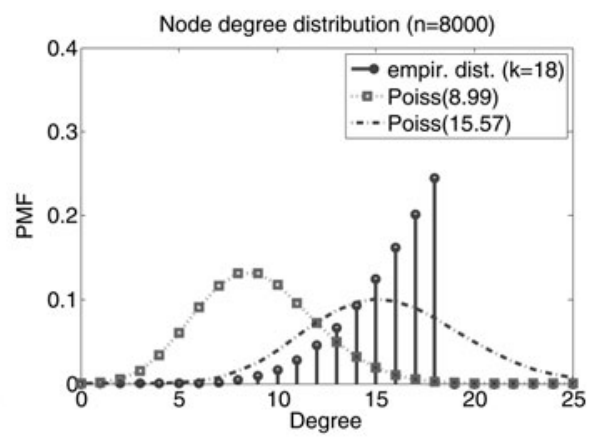

(b)

Figure 6 Node degree distribution and Poisson distribution approximation for (a) $n=200$ and $k=11$ and (b) $n=8000$ and $k=18$. 
very small, especially for $n=8000$. This is a consequence of the well-known concentration of a Poisson rv around its mean.

\section{CONCLUSIONS}

We have proposed a new random graph model that allows us to capture the strategic nature of nodes, which arises naturally in many situations. The proposed random graphs are constructed in a very different fashion than are existing random graph models and likely exhibit different statistical behavior. We believe that the new random graph model will prove to be useful for modeling the network formation in distributed systems in which the agents are free to choose their neighbors based on the estimated benefits and/or local information.

We are currently working to generalize the proposed random graph model. First, we are studying the case in which the edge values are not symmetric, but instead are correlated. Second, we are also examining scenarios in which the number of potential edges chosen by each node varies and is modeled as a random variable.

\section{ACKNOWLEDGMENTS}

The authors would like to thank Professors Armand M. Makowski and Vijay G. Subramanian for many helpful discussions and anonymous reviewers for many constructive comments.

\section{FUNDING}

This work was supported in part by the National Science Foundation under Grant CCF 08-30675 and a grant from National Institute of Standards and Technology (NIST).

\section{APPENDICES}

\section{Appendix A. Proof of Theorem 5.1}

First, note that the probability of graph connectivity is increasing in $k$. Therefore, in order to prove the theorem, it suffices to show that when $k(n)=\beta \log (n), n \in \mathbb{N}_{+}$, for any $\beta>\beta^{\star},{ }^{4}$ the probability that $\mathbb{G}(n, k(n))$ is connected goes to one as $n$ increases. For each $n \in \mathbb{N}_{+}$, we first order the edge values seen by each node by decreasing value: For each $i \in \mathcal{V}^{(n)}$, let $U_{i, 1}^{(n)}, \ldots, U_{i, n-1}^{(n)}$ denote the order statistics of $V_{i, j}, j \in \mathcal{V}^{(n)} \backslash\{i\}$. ${ }^{5}$ Then, it is clear that $U_{i, 1}^{(n)}, \ldots, U_{i, k(n)}^{(n)}$ are the values of the $k(n)$ edges selected by node $i$.

Recall from observation O-3 in Section 2 that we can assume any continuous distribution for the edge value distribution $F$ without affecting our result. Taking advantage of this fact, we assume $F \sim \operatorname{exponential(1)~in~the~proof.~In~other~words,~edge~values~} V_{i, j}, i \neq j$, are assumed to be i.i.d. exponential rvs with a mean of one.

\footnotetext{
${ }^{4}$ When $\beta \log (n)$ is not an integer, we can assume that $k(n)=\lfloor\beta \log (n)\rfloor$ without any problem because we can always find $\underline{\beta}$ satisfying $\beta^{\star}<\underline{\beta}<\beta$ and finite $n^{\star}(\underline{\beta})$ such that, for all $n \geq n^{\star}(\underline{\beta})$, we have $\beta \log (n) \leq\lfloor\beta \log (n)\rfloor$.

${ }^{5}$ Throughout the proofs, we assume that the order statistics are ordered by decreasing value.
} 
We first introduce a lemma that will be used to complete the proof of the theorem. Its proof is given in Appendix B.

Lemma A.1. Suppose that the edge values are given by i.i.d. exponential(1) rvs. For each $n \in \mathbb{N}_{+}$and $i \in \mathcal{V}^{(n)}$, let

$$
A_{i}^{(n)}=\left\{\left(U_{i, k(n)}^{(n)}-\log \left(\frac{n-1}{k(n)}\right)\right) \in\left(-\sqrt{\frac{2}{\beta}}, \sqrt{\frac{2}{\beta}}\right)\right\}
$$

and $A^{(n)}:=\bigcap_{i \in \mathcal{V}^{(n)}} A_{i}^{(n)}$. Then, $\mathbb{P}\left[A^{(n)}\right] \rightarrow 1$ as $n \rightarrow \infty$.

Lemma A.1 tells us that, as $n$ increases, whp

$$
U_{i, k(n)}^{(n)} \in\left(\log \left(\frac{n-1}{k(n)}\right)-\sqrt{\frac{2}{\beta}}, \log \left(\frac{n-1}{k(n)}\right)+\sqrt{\frac{2}{\beta}}\right)=: B^{\star}(n)
$$

simultaneously for all $i \in \mathcal{V}^{(n)}$.

For each $n \in \mathbb{N}_{+}$, let $\bar{v}_{n}:=\log ((n-1) / k(n))+\sqrt{2 / \beta}$ and

$$
p^{*}(n):=\mathbb{P}\left[V_{1,2}>\bar{v}_{n}\right]=e^{-(\log (n-1)-\log (k(n))+\sqrt{2 / \beta})}=\frac{k(n)}{n-1} e^{-\sqrt{2 / \beta}} .
$$

Substituting $\beta \log (n)$ for $k(n)$,

$$
p^{*}(n)=\frac{\beta \log (n)}{n-1} e^{-\sqrt{2 / \beta}}=\zeta \frac{\log (n)}{n-1},
$$

where $\zeta:=\beta \exp (-\sqrt{2 / \beta})$. From the assumption $\beta>\beta^{\star}=\exp \left(\sqrt{2 / \beta^{\star}}\right)>\exp (\sqrt{2 / \beta})$, we have $\zeta>1$.

We complete the proof with the help of new random graphs that are constructed using the same vertex sets and edge values used to define $\mathbb{G}(n, k(n)), n \in \mathbb{N}_{+}$: For each $n \in \mathbb{N}_{+}$, define a new random graph $\tilde{G}\left(n, \bar{v}_{n}\right)=\left(\mathcal{V}^{(n)}, \mathcal{E}_{\text {new }}^{(n)}\right)$, where

$$
\mathcal{E}_{\text {new }}^{(n)}=\left\{(i, j) \mid i, j \in \mathcal{V}^{(n)}, i \neq j, V_{i, j}>\bar{v}_{n}\right\} .
$$

From the i.i.d. assumption of the edge values and (A.1),

$$
\mathbb{P}\left[(i, j) \in \mathcal{E}_{\text {new }}^{(n)}\right]=\zeta \frac{\log (n)}{n-1}=p^{*}(n) \text { for all } i, j \in \mathcal{V}^{(n)}, i \neq j,
$$

independently of other edges. In other words, each undirected edge $(i, j)$ between a pair of nodes $i$ and $j$ belongs to $\mathcal{E}_{\text {new }}^{(n)}$ with probability $p^{*}(n)$, independently of each other. Therefore, the new random graph $\tilde{G}\left(n, \bar{v}_{n}\right)$ is in fact an Erdös-Rényi graph $G\left(n, p^{*}(n)\right)$, constructed using the same edge values, with the edge probability $p^{*}(n)$ given in (A.1). Moreover, since $\zeta>1[19,20]$, we know

$$
\mathbb{P}\left[\tilde{G}\left(n, \bar{v}_{n}\right) \text { is connected }\right] \rightarrow 1 \text { as } n \rightarrow \infty .
$$

We now upper bound the probability that $\mathbb{G}(n, k(n))$ is not connected, using the law of total probability.

$\mathbb{P}[\mathbb{G}(n, k(n))$ is not connected $]=\mathbb{P}\left[\left(A^{(n)}\right)^{c} \cap\{\mathbb{G}(n, k(n))\right.$ is not connected $\left.\}\right]$ 


$$
\begin{aligned}
& +\mathbb{P}\left[A^{(n)} \cap\{\mathbb{G}(n, k(n)) \text { is not connected }\}\right] \\
\leq & \mathbb{P}\left[\left(A^{(n)}\right)^{c}\right]+\mathbb{P}\left[A^{(n)} \cap\{\mathbb{G}(n, k(n)) \text { is not connected }\}\right]
\end{aligned}
$$

First, Lemma A.1 states that $\mathbb{P}\left[\left(A^{(n)}\right)^{c}\right]$ goes to zero as $n$ increases. Second, when the event $A^{(n)}$ is true, $U_{i, k(n)}^{(n)} \in B^{\star}(n)$ and, consequently, $U_{i, k(n)}^{(n)}<\bar{v}_{n}$ for all $i \in \mathcal{V}^{(n)}$. This implies that all edges $(i, j), i, j \in \mathcal{V}^{(n)}$ and $i \neq j$, with $V_{i, j}>\bar{v}_{n}$ belong to the edge set $\mathcal{E}^{(n)}$ of $\mathbb{G}(n, k(n))$ because both of the end nodes would choose the edges. Hence, since the edges in $\tilde{G}\left(n, \bar{v}_{n}\right)$ are precisely those with edge values larger than $\bar{v}_{n}$, when the event $A^{(n)}$ occurs, all the edges in $\mathcal{E}_{\text {new }}^{(n)}$ also belong to $\mathcal{E}^{(n)}$. This in turn tells us that when (a) the event $A^{(n)}$ happens and (b) the graph $\tilde{G}\left(n, \bar{v}_{n}\right)$ is connected, the graph $\mathbb{G}(n, k(n))$ is also connected. in (A.3).

Based on this observation, we can find the following upper bound for the second term

$$
\begin{aligned}
\mathbb{P}\left[A^{(n)} \cap\{\mathbb{G}(n, k(n)) \text { is not connected }\}\right] & \leq \mathbb{P}\left[A^{(n)} \cap\left\{\tilde{G}\left(n, \bar{v}_{n}\right) \text { is not connected }\right\}\right] \\
& \leq \mathbb{P}\left[\tilde{G}\left(n, \bar{v}_{n}\right) \text { is not connected }\right]
\end{aligned}
$$

From (A.2), as $n$ increases, the random graph $\tilde{G}\left(n, \bar{v}_{n}\right)$ is connected with probability approaching one, thereby implying that (A.4) goes to zero as $n \rightarrow \infty$. Therefore, the second term in (A.3) goes to zero as $n$ increases, and $\mathbb{P}[\mathbb{G}(n, k(n))$ is not connected] $\rightarrow$ 0 as $n \rightarrow \infty$.

\section{Appendix B. Proof of Lemma A.1}

Define $B(n):=(-\sqrt{2 \log (n)}, \sqrt{2 \log (n)}), n \in \mathbb{N}_{+}$. From [22, (2.12), p. 24] and [14, Theorem 10.8 .1$, p. 312], for any $\delta>0$, there exists finite $n(\delta)$ such that, for all $n \geq n(\delta)$,

$$
\begin{aligned}
\mathbb{P}\left[\sqrt{k(n)}\left(U_{i, k(n)}^{(n)}-\log ((n-1) / k(n))\right) \notin B(n)\right] & =\mathbb{P}\left[\left(A_{i}^{(n)}\right)^{c}\right] \\
& \leq 2(1+\delta) Q(\sqrt{2 \log (n)})
\end{aligned}
$$

where $Q$ is the complementary cumulative distribution function of a standard normal rv. Using a well-known upper bound for $Q(x), \exp \left(-x^{2} / 2\right) /(\sqrt{2 \pi} x)$ [30],

$$
\begin{aligned}
\mathbb{P}\left[\left(A_{i}^{(n)}\right)^{c}\right] & \leq 2(1+\delta) \frac{1}{\sqrt{2 \pi}} \frac{\exp \left(-\frac{2 \log (n)}{2}\right)}{\sqrt{2 \log (n)}} \\
& =(1+\delta) \frac{\exp (-\log (n))}{\sqrt{\pi \log (n)}}=\frac{1+\delta}{n \sqrt{\pi \log (n)}} .
\end{aligned}
$$

Since $A^{(n)}=\bigcap_{i \in \mathcal{V}^{(n)}} A_{i}^{(n)}$, using a union bound and (A.5),

$$
\mathbb{P}\left[\left(A^{(n)}\right)^{c}\right] \leq \sum_{i \in \mathcal{V}^{(n)}} \mathbb{P}\left[\left(A_{i}^{(n)}\right)^{c}\right] \leq \frac{1+\delta}{\sqrt{\pi \log (n)}} .
$$

Therefore, $\mathbb{P}\left[\left(A^{(n)}\right)^{c}\right] \rightarrow 0$ or, equivalently, $\mathbb{P}\left[A^{(n)}\right] \rightarrow 1$ as $n \rightarrow \infty$. 


\section{Appendix C. Proof of Theorem 5.2}

Note that, for any fixed $n \geq 2$, the probability $\mathbb{P}[\mathbb{G}(n, k)]$ contains at least one isolated node is decreasing in $k$. Hence, if Theorem 5.2 is true with $k(n)=\alpha \log (n)$ for some $\alpha<0.5,{ }^{6}$ then it is also true when $k(n) \leq \alpha \log (n)$. For this reason, in order to prove the theorem, it suffices to show that the claim in Theorem 5.2 holds if $k(n)=\alpha \log (n)$ with $\alpha<0.5$.

To this end, we use the (second) moment method: Define $\mathbb{Z}_{+}:=\{0,1,2, \ldots\}$ to be the set of nonnegative integers. Suppose $\left\{Z_{n} ; n=1,2, \ldots\right\}$ is a sequence of $\mathbb{Z}_{+}$-valued rvs with finite second moment, i.e., $\mathbb{E}\left[Z_{n}^{2}\right]<\infty$ for all $n \in \mathbb{N}$. Then,

$$
\lim _{n \rightarrow \infty} \mathbb{P}\left[Z_{n}=0\right]=0 \text { if } \lim _{n \rightarrow \infty} \frac{\left(\mathbb{E}\left[Z_{n}\right]\right)^{2}}{\mathbb{E}\left[Z_{n}^{2}\right]}=1 .
$$

Equation (A.6) can be easily proved using Cauchy-Schwarz inequality [26, p. 65].

For each $n \in \mathbb{N}_{+}$, define $I_{i}^{(n)}=\mathbf{1}$ \{node $i$ is isolated in $\mathbb{G}(n, k(n))$ \}, $i \in \mathcal{V}^{(n)}$, and $C^{(n)}=\sum_{i=1}^{n} I_{i}^{(n)}$. Clearly, $C^{(n)}$ denotes the total number of isolated nodes in $\mathbb{G}(n, k(n))$. We prove that $\mathbb{P}\left[C^{(n)}=0\right] \rightarrow 0$ as $n \rightarrow \infty$ by means of (A.6). To this end, we first rewrite both $\left(\mathbb{E}\left[C^{(n)}\right]\right)^{2}$ and $\left.\mathbb{E}\left[\left(C^{(n)}\right)\right)^{2}\right]$ in a more convenient form and then prove that their ratio converges to one with increasing $n$, i.e., $\left.\lim _{n \rightarrow \infty}\left(\mathbb{E}\left[C^{(n)}\right]\right)^{2} / \mathbb{E}\left[\left(C^{(n)}\right)\right)^{2}\right]=1$.

Because $I_{i}^{(n)}, i \in \mathcal{V}^{(n)}$, are exchangeable, we have

$$
\mathbb{E}\left[C^{(n)}\right]=n \mathbb{E}\left[I_{1}^{(n)}\right]
$$

and

$$
\mathbb{E}\left[\left(C^{(n)}\right)^{2}\right]=\mathbb{E}\left[\left(\sum_{i=1}^{n} I_{i}^{(n)}\right)^{2}\right]=n \mathbb{E}\left[I_{1}^{(n)}\right]+n(n-1) \mathbb{E}\left[I_{1}^{(n)} \cdot I_{2}^{(n)}\right] .
$$

Using (A.7) and (A.8), we get

$$
\begin{aligned}
& \frac{\left(\mathbb{E}\left[C^{(n)}\right]\right)^{2}}{\mathbb{E}\left[\left(C^{(n)}\right)^{2}\right]}=\frac{\left(n \mathbb{E}\left[I_{1}^{(n)}\right]\right)^{2}}{n \mathbb{E}\left[I_{1}^{(n)}\right]+n(n-1) \mathbb{E}\left[I_{1}^{(n)} I_{2}^{(n)}\right]} \\
& \quad=\left(\left(n \mathbb{E}\left[I_{1}^{(n)}\right]\right)^{-1}+\frac{n-1}{n} \cdot \frac{\mathbb{E}\left[I_{1}^{(n)} I_{2}^{(n)}\right]}{\left(\mathbb{E}\left[I_{1}^{(n)}\right]\right)^{2}}\right)^{-1} .
\end{aligned}
$$

The proof of the theorem can be completed with help of Lemmas A.2 and A.3.

Lemma A.2. Under the condition in Theorem 5.2, the expected values of $C^{(n)}, n \in \mathbb{N}_{+}$, satisfy

$$
\lim _{n \rightarrow \infty} \mathbb{E}\left[C^{(n)}\right]=\lim _{n \rightarrow \infty}\left(n \mathbb{E}\left[I_{1}^{(n)}\right]\right)=\infty
$$

${ }^{6}$ For a similar reason stated in the proof of Theorem 5.1 in Appendix A, when $\alpha \log (n)$ is not an integer, we can assume that $k(n)=\lceil\alpha \log (n)\rceil$. 
Proof. Please see Appendix D for a proof.

Lemma A.3. Suppose that the condition in Theorem 5.2 holds. Then,

$$
\limsup _{n \rightarrow \infty} \frac{\mathbb{E}\left[I_{1}^{(n)} I_{2}^{(n)}\right]}{\left(\mathbb{E}\left[I_{1}^{(n)}\right]\right)^{2}} \leq 1 .
$$

Proof. Please see Appendix E for a proof.

Lemmas A.2 and A.3, together with (A.9) and the inequality $\left(\mathbb{E}\left[C^{(n)}\right]\right)^{2} \leq \mathbb{E}\left[\left(C^{(n)}\right)^{2}\right]$, imply that $\lim _{n \rightarrow \infty}\left(\left(\mathbb{E}\left[C^{(n)}\right]\right)^{2} / \mathbb{E}\left[\left(C^{(n)}\right)^{2}\right]\right)=1$. Therefore, (A.6) tells us that $\mathbb{P}\left[C^{(n)}=\right.$ $0] \rightarrow 0$ as $n \rightarrow \infty$, and the probability that $\mathbb{G}(n, k(n))$ has at least one isolated node converges to one, completing the proof of the theorem.

In addition, these lemmas also tell us

$$
\lim _{n \rightarrow \infty} \frac{\mathbb{E}\left[I_{1}^{(n)} I_{2}^{(n)}\right]}{\left(\mathbb{E}\left[I_{1}^{(n)}\right]\right)^{2}}=1 .
$$

This can be easily proved by contradiction: Suppose that (A.10) is not true and

$$
\liminf _{n \rightarrow \infty} \frac{\mathbb{E}\left[I_{1}^{(n)} I_{2}^{(n)}\right]}{\left(\mathbb{E}\left[I_{1}^{(n)}\right]\right)^{2}}<1 .
$$

This inequality, together with (A.9) and Lemma A.2, implies

$$
\limsup _{n \rightarrow \infty} \frac{\left(\mathbb{E}\left[C^{(n)}\right]\right)^{2}}{\mathbb{E}\left[\left(C^{(n)}\right)^{2}\right]}>1,
$$

which is a contradiction.

\section{Appendix D. Proof of Lemma A.2}

We prove the lemma by showing that, for any fixed $\xi \in(\alpha, 1)$, we have $\mathbb{E}\left[I_{1}^{(n)}\right]=$ $\Omega\left(n^{-\xi}\right)$. This means $\mathbb{E}\left[C^{(n)}\right]=n \mathbb{E}\left[I_{1}^{(n)}\right]=\Omega\left(n^{1-\xi}\right)$ with $1-\xi>0$. Making use of observation O-3 in Section 2, we prove this with $F \sim \operatorname{exponential(1).~}$

Define $N_{1}^{(n)}=\left\{j \in \mathcal{V}^{(n)} \backslash\{1\} \mid(1, j) \in E_{1}^{(n)}\right\}$ to be the set of potential neighbors selected by node 1 . In order to prove $\mathbb{E}\left[I_{1}^{(n)}\right]=\mathbb{P}\left[I_{1}^{(n)}=1\right]=\Omega\left(n^{-\xi}\right)$, we consider lower bounds for $\mathbb{E}\left[I_{1}^{(n)}\right]$ and demonstrate that the lower bounds are $\Omega\left(n^{-\xi}\right)$. These lower bounds are constructed using the events that (i) $U_{1,1}^{(n)}$ is smaller than some given threshold, and (ii) $U_{i, k(n)}^{(n)}$ is greater than the threshold for all $i \in N_{1}^{(n)}$. When these events are true, we have $U_{1,1}^{(n)}<U_{i, k(n)}^{(n)}$ for all $i \in N_{1}^{(n)}$ and no node in $N_{1}^{(n)}$ chooses an edge with node 1 , hence, implying that node 1 is isolated. 
Pick $\gamma \in(0.5,1)$ and, for each $n \in \mathbb{N}_{+}$, let $\Lambda_{\gamma}(n):=\left(-\phi_{\gamma}(n), \phi_{\gamma}(n)\right)$, where

$$
\phi_{\gamma}(n):=\sqrt{\frac{4 \gamma \log (\log (n))}{k(n)}} .
$$

For every $i \in \mathcal{V}^{(n)}$, let

$$
\tilde{A}_{i}^{(n)}(\gamma)=\left\{\left(U_{i, k(n)}^{(n)}-\log ((n-1) / k(n))\right) \notin \Lambda_{\gamma}(n)\right\},
$$

and $\tilde{A}^{(n)}(\gamma):=\bigcup_{i \in N_{1}^{(n)}} \tilde{A}_{i}^{(n)}(\gamma)$. In addition, define

$$
\tilde{B}_{1}^{(n)}(\gamma)=\left\{\left(U_{1,1}^{(n)}-\log ((n-1) / k(n))\right)<-\phi_{\gamma}(n)\right\} .
$$

Using these events, we can find the following lower bound for $\mathbb{E}\left[I_{1}^{(n)}\right]$.

$$
\begin{aligned}
\mathbb{E}\left[I_{1}^{(n)}\right] & =\mathbb{P}\left[I_{1}^{(n)}=1\right] \geq \mathbb{P}\left[\left(\tilde{A}^{(n)}(\gamma)\right)^{c} \cap\left\{I_{1}^{(n)}=1\right\}\right] \\
& \geq \mathbb{P}\left[\left(\tilde{A}^{(n)}(\gamma)\right)^{c} \cap \tilde{B}_{1}^{(n)}(\gamma)\right] \\
& =\mathbb{P}\left[\left(\tilde{A}^{(n)}(\gamma)\right)^{c} \mid \tilde{B}_{1}^{(n)}(\gamma)\right] \mathbb{P}\left[\tilde{B}_{1}^{(n)}(\gamma)\right],
\end{aligned}
$$

where the second inequality in (A.13) is a result of the following fact: first, in the event $\left(\tilde{A}^{(n)}(\gamma)\right)^{c}$,

$$
\left(U_{i, k(n)}^{(n)}-\log ((n-1) / k(n))\right) \in \Lambda_{\gamma}(n) \text { for all } i \in N_{1}^{(n)} .
$$

Thus, nodes in $N_{1}^{(n)}$ do not pick any edge whose value is less than $\log ((n-1) / k(n))-\phi_{\gamma}(n)$.

Second, as $U_{1,1}^{(n)}=\max \left\{V_{1, i}, \quad i \in \mathcal{V}^{(n)} \backslash\{1\}\right\}$ is the largest edge value seen by node 1 , together with the assumed symmetry of edge values, event $\tilde{B}_{1}^{(n)}(\gamma)$ implies $V_{i, 1}<$ $\log ((n-1) / k(n))-\phi_{\gamma}(n)$ for all $i \in \mathcal{V}^{(n)} \backslash\{1\}$. As a result, when $\left(\tilde{A}^{(n)}(\gamma)\right)^{c} \cap \tilde{B}_{1}^{(n)}(\gamma)$ occurs, we have $V_{i, 1}<U_{i, k(n)}^{(n)}$ for all $i \in N_{1}^{(n)}$ and node 1 is isolated. This tells us $\mathbb{P}\left[\left(\tilde{A}^{(n)}(\gamma)\right)^{c} \cap \tilde{B}_{1}^{(n)}(\gamma)\right] \leq \mathbb{P}\left[\left(\tilde{A}^{(n)}(\gamma)\right)^{c} \cap\left\{I_{1}^{(n)}=1\right\}\right]$, proving the inequality in (A.13).

In light of (A.14), Lemma A.2 is an immediate consequence of the following two claims we prove.

C1-a. $\mathbb{P}\left[\tilde{B}_{1}^{(n)}(\gamma)\right]=\Omega\left(n^{-\xi}\right)$ for any $\xi \in(\alpha, 1)$; and

C1-b. $\lim _{n \rightarrow \infty} \mathbb{P}\left[\left(\tilde{A}^{(n)}(\gamma)\right)^{c} \mid \tilde{B}_{1}^{(n)}(\gamma)\right]=1$.

\section{D.1. Proof of claim C1-a}

First, since $U_{1,1}^{(n)}$ is the maximum among $n-1$ i.i.d. exponential(1) rvs, the distribution of $\left(U_{1,1}^{(n)}-\log (n-1)\right)$ converges to a standard Gumbel distribution $[14,15]$, i.e., for all $x \in \mathbb{R}$,

$$
\mathbb{P}\left[\left(U_{1,1}^{(n)}-\log (n-1)\right) \leq x\right] \rightarrow \exp (-\exp (-x)) \text { as } n \rightarrow \infty
$$


Using this distributional convergence and the definition of event $\tilde{B}_{1}^{(n)}(\gamma)$ in (A.12), after a little algebra, we can prove

$$
\mathbb{P}\left[\tilde{B}_{1}^{(n)}(\gamma)\right] \sim \exp \left(-\exp \left(-\left(-\log (k(n))-\phi_{\gamma}(n)\right)\right)\right)
$$

Substituting $\alpha \log (n)$ for $k(n)$,

$$
\mathbb{P}\left[\tilde{B}_{1}^{(n)}(\gamma)\right] \sim \exp \left(-\alpha \log (n) \exp \left(\phi_{\gamma}(n)\right)\right)=\frac{1}{n^{\alpha} \psi(n)},
$$

where $\psi(n):=\exp \left(\phi_{\gamma}(n)\right)$. Note from (A.11) that $\phi_{\gamma}(n) \rightarrow 0$ as $n \rightarrow \infty$. Thus, $\lim _{n \rightarrow \infty} \psi(n)=1$ and, for any given $\xi \in(\alpha, 1)$, we can find finite $n^{\star}(\xi)$ such that, for all $n \geq n^{\star}(\xi)$, we have $\alpha \psi(n)<\xi$. Together with (A.15), this implies $\mathbb{P}\left[\tilde{B}_{1}^{(n)}(\gamma)\right]=\Omega\left(n^{-\xi}\right)$.

\section{2. Proof of claim C1-b}

In order to prove the claim, we show $\lim _{n \rightarrow \infty} \mathbb{P}\left[\tilde{A}^{(n)}(\gamma) \mid \tilde{B}_{1}^{(n)}(\gamma)\right]=0$. To this end, we construct upper bounds for $\mathbb{P}\left[\tilde{A}^{(n)}(\gamma) \mid \tilde{B}_{1}^{(n)}(\gamma)\right]$ with the help of another sequence of events (denoted by $\bar{A}^{(n)}, n \in \mathbb{N}_{+}$) that are closely related to $\tilde{A}^{(n)}(\gamma)$ and are independent of $\tilde{B}_{1}^{(n)}(\gamma)$, and demonstrate that these upper bounds converge to zero as $n \rightarrow \infty$.

Recall that, in the event $\tilde{B}_{1}^{(n)}(\gamma)$, we have

$$
V_{i, 1} \leq U_{1,1}^{(n)}<\log ((n-1) / k(n))-\phi_{\gamma}(n) \text { for all } i \in \mathcal{V}^{(n)} \backslash\{1\}
$$

Clearly, conditional on $\tilde{B}_{1}^{(n)}(\gamma)$, the event $\tilde{A}_{i}^{(n)}(\gamma), i \in \mathcal{V}^{(n)} \backslash\{1\}$, takes place if and only if the $k(n)$-th largest value among the remaining edge values $V_{i, j}, j \in \mathcal{V}^{(n)} \backslash\{1, i\}$, does not belong to $\Lambda_{\gamma}(n)$.

Let $\tilde{U}_{i, \ell}^{(n)}, \ell=1,2, \ldots, n-2$, be the order statistics of $V_{i, j}, j \in \mathcal{V}^{(n)} \backslash\{1, i\}$. We denote the event $\tilde{A}_{i}^{(n)}(\gamma)$ conditional on $\tilde{B}_{1}^{(n)}(\gamma)$ by

$$
\begin{aligned}
\tilde{A}_{i}^{(n)}(\gamma) \mid \tilde{B}_{1}^{(n)}(\gamma) & =\left\{\left(\tilde{U}_{i, k(n)}^{(n)}-\log ((n-1) / k(n))\right) \notin \Lambda_{\gamma}(n)\right\} \mid \tilde{B}_{1}^{(n)}(\gamma) \\
& =\left\{\left(\tilde{U}_{i, k(n)}^{(n)}-\log ((n-2) / k(n))\right) \notin \Lambda_{\gamma}^{\star}(n)\right\} \mid \tilde{B}_{1}^{(n)}(\gamma),
\end{aligned}
$$

where

$$
\Lambda_{\gamma}^{\star}(n):=\left(-\phi_{\gamma}(n)+\log \left(\frac{n-1}{n-2}\right), \phi_{\gamma}(n)+\log \left(\frac{n-1}{n-2}\right)\right) .
$$

Because $\log ((n-1) /(n-2))=o(1 / \sqrt{\log (n)})$ and $\gamma \in(0.5,1)$, there exists finite $\bar{n}(\gamma)$ such that, for all $n \geq \bar{n}(\gamma)$,

$$
\bar{\Lambda}(n):=\left(-\sqrt{\frac{2 \log (\log (n))}{k(n)}}, \sqrt{\frac{2 \log (\log (n))}{k(n)}}\right) \subseteq \Lambda_{\gamma}^{\star}(n) .
$$

Define

$$
\bar{A}_{i}^{(n)}=\left\{\left(\tilde{U}_{i, k(n)}-\log ((n-2) / k(n))\right) \notin \bar{\Lambda}(n)\right\}
$$


and $\bar{A}^{(n)}:=\bigcup_{i \in N_{1}^{(n)}} \bar{A}_{i}^{(n)}$. For all $n \geq \bar{n}(\gamma)$, because $\bar{\Lambda}(n) \subseteq \Lambda_{\gamma}^{\star}(n)$, we have

$$
\mathbb{P}\left[\tilde{A}^{(n)}(\gamma) \mid \tilde{B}_{1}^{(n)}(\gamma)\right] \leq \mathbb{P}\left[\bar{A}^{(n)} \mid \tilde{B}_{1}^{(n)}(\gamma)\right]=\mathbb{P}\left[\bar{A}^{(n)}\right],
$$

where the equality follows from the assumed independence of edge values.

We now prove $\mathbb{P}\left[\bar{A}^{(n)}\right] \rightarrow 0$ as $n \rightarrow \infty$. Following similar steps as in the proof of Lemma A.1 in Appendix B, we can show that, for any $\delta>0$ and all sufficiently large $n$,

$$
\begin{aligned}
\mathbb{P}\left[\bar{A}_{i}^{(n)}\right] & \leq 2(1+\delta) Q(\sqrt{2 \log (\log (n))}) \leq(1+\delta) \frac{\exp (-\log (\log (n)))}{\sqrt{\pi \log (\log (n))}} \\
& =\frac{1+\delta}{\log (n) \sqrt{\pi \log (\log (n))}} .
\end{aligned}
$$

Using a union bound and (A.16) and substituting $\alpha \log (n)$ for $k(n)=\left|N_{1}^{(n)}\right|$, we obtain

$$
\mathbb{P}\left[\bar{A}^{(n)}\right] \leq \sum_{i \in N_{1}^{(n)}} \mathbb{P}\left[\bar{A}_{i}^{(n)}\right]=\frac{\alpha(1+\delta)}{\sqrt{\pi \log (\log (n))}} \rightarrow 0 \text { as } n \rightarrow \infty .
$$

Together with the inequality $\mathbb{P}\left[\tilde{A}^{(n)}(\gamma) \mid \tilde{B}_{1}^{(n)}(\gamma)\right] \leq \mathbb{P}\left[\bar{A}^{(n)}\right]$, (A.17) tells us

$$
\lim _{n \rightarrow \infty} \mathbb{P}\left[\left(\tilde{A}^{(n)}(\gamma)\right)^{c} \mid \tilde{B}_{1}^{(n)}(\gamma)\right]=1 .
$$

\section{Appendix E. Proof of Lemma A.3}

In order to prove the lemma, we first demonstrate

$$
\mathbb{P}\left[I_{1}^{(n)}=1, I_{2}^{(n)}=1\right] \leq\left(\mathbb{P}\left[I_{1}^{(n)}=1\right]\right)^{2}+o\left(\left(\mathbb{P}\left[I_{1}^{(n)}=1\right]\right)^{2}\right) .
$$

This inequality can be easily shown with the help of Theorem (3) in [16] as follows.

First, recall from the proof of Lemma A.2 in Appendix D that $\mathbb{P}\left[I_{1}^{(n)}=1\right]=\Omega\left(n^{-\xi}\right)$ for any $\xi \in(\alpha, 1)$. Let $\Delta:=0.5-\alpha>0$ and $\xi^{\dagger}:=0.5(1-\Delta)=\alpha+0.5 \Delta \in(\alpha, 1)$. Hence, $\mathbb{P}\left[I_{1}^{(n)}=1\right]=\Omega\left(n^{-\xi^{\dagger}}\right)$ or, equivalently,

$$
\left(\mathbb{P}\left[I_{1}^{(n)}=1\right]\right)^{2}=\Omega\left(n^{-(1-\Delta)}\right)=\omega\left(n^{-1}\right) .
$$

Second, because $I_{1}^{(n)}, i \in \mathcal{V}^{(n)}$, are exchangeable, Theorem (3) of [16], which is a generalization of de Finetti's theorem for finite exchangeable sequences, tells us

$$
\mathbb{P}\left[I_{1}^{(n)}=1, I_{2}^{(n)}=1\right] \leq \mathbb{P}\left[I_{1}^{(n)}=1\right] \mathbb{P}\left[I_{2}^{(n)}=1\right]+\frac{8}{n} .
$$

Since $\left(\mathbb{P}\left[I_{1}^{(n)}=1\right]\right)^{2}=\omega\left(n^{-1}\right)$ from (A.19), we have

$$
\frac{8}{n}=o\left(\left(\mathbb{P}\left[I_{1}^{(n)}=1\right]\right)^{2}\right)
$$


This completes the proof of (A.18).

Using the upper bound in (A.20) for the numerator, we obtain the following.

$$
\begin{aligned}
\limsup _{n \rightarrow \infty} \frac{\mathbb{P}\left[I_{1}^{(n)}=1, I_{2}^{(n)}=1\right]}{\left(\mathbb{P}\left[I_{1}^{(n)}=1\right]\right)^{2}} & \leq \limsup _{n \rightarrow \infty} \frac{\left(\mathbb{P}\left[I_{1}^{(n)}=1\right]\right)^{2}+8 / n}{\left(\mathbb{P}\left[I_{1}^{(n)}=1\right]\right)^{2}} \\
& =1+\limsup _{n \rightarrow \infty} \frac{8 / n}{\left(\mathbb{P}\left[I_{1}^{(n)}=1\right]\right)^{2}}
\end{aligned}
$$

By (A.21), the second term in (A.22) is equal to zero and, consequently, we get the desired inequality in the lemma.

\section{Appendix F. Proof of Theorem 5.3}

Theorem 5.3 can be proved with the help of the following result for Erdös-Rényi random graphs.

Theorem 2.8.6. [17, p. 67]: Suppose that the edge selection probabilities $p(n), n \in$ $\mathbb{N}_{+}$, of Erdös-Rényi random graphs $G(n, p(n))$ satisfy $(i) \liminf _{n \rightarrow \infty} n p(n) / \log (n)>1$ and (ii) $\lim _{n \rightarrow \infty}(\log (n p(n)) / \log (n))=0$. Then, the diameters of the Erdös-Rényi random graphs $G(n, p(n))$ satisfy

$$
\mathbb{P}\left[\left|\frac{D(G(n, p(n)))}{\log (n) / \log (n p(n))}-1\right|>\epsilon\right] \rightarrow 0 \text { as } n \rightarrow \infty
$$

for all $\epsilon>0$.

Let

$$
\underline{\kappa}=\liminf _{n \rightarrow \infty} \frac{k(n)}{\log (n)} \text { and } \bar{\kappa}=\limsup _{n \rightarrow \infty} \frac{k(n)}{\log (n)} .
$$

Then, for any $\epsilon>0$, there exists finite $\tilde{n}(\epsilon)$ such that, for all $n \geq \tilde{n}(\epsilon)$, we have $k(n) \epsilon$ $[(\underline{\kappa}-\epsilon) \log (n),(\bar{\kappa}+\epsilon) \log (n)]$. Pick $\epsilon$ satisfying $0<\epsilon<\underline{\kappa}-\beta^{\star}$, and let $\tilde{n}(\epsilon)$ be the smallest $n \in \mathbb{N}_{+}$that satisfies the condition.

In the rest of the proof, utilizing observation O-3 in Section 2, we assume $F \sim$ exponential(1). For $n \geq \tilde{n}(\epsilon)$, let $\kappa(n):=k(n) / \log (n) \in[\underline{\kappa}-\epsilon, \bar{\kappa}+\epsilon]$. Define

$$
p_{1}(n)=\mathbb{P}\left[V_{1,2}>\log \left(\frac{n-1}{k(n)}\right)+\sqrt{\frac{2}{\kappa(n)}}\right]=\zeta_{1}(n) \frac{\log (n)}{n-1},
$$

where $\zeta_{1}(n):=\kappa(n) e^{-\sqrt{2 / \kappa(n)}}>1$ because $\kappa(n) \geq \underline{\kappa}-\epsilon>\beta^{\star}$, and

$$
p_{2}(n)=\mathbb{P}\left[V_{1,2}>\log \left(\frac{n-1}{k(n)}\right)-\sqrt{\frac{2}{\kappa(n)}}\right]=\zeta_{2}(n) \frac{\log (n)}{n-1},
$$

where $\zeta_{2}(n):=\kappa(n) e^{\sqrt{2 / \kappa(n)}}>\zeta_{1}(n)$. 
For $n \in \mathbb{N}_{+}$, denote by $\mathcal{E}_{E R, 1}^{(n)}$ (resp. $\left.\mathcal{E}_{E R, 2}^{(n)}\right)$ the set of edges $(i, j), i, j \in \mathcal{V}^{(n)}(i \neq j)$, satisfying $V_{i, j}>\log ((n-1) / k(n))+\sqrt{2 / \kappa(n)}\left(\operatorname{resp} . V_{i, j}>\log ((n-1) / k(n))-\sqrt{2 / \kappa(n)}\right)$. Let $G\left(n, p_{\ell}(n)\right):=\left(\mathcal{V}^{(n)}, \mathcal{E}_{E R, \ell}^{(n)}\right), \ell=1,2$. As explained in Appendix A, $G\left(n, p_{\ell}(n)\right)$, $\ell=1,2$, are Erdös-Rényi random graphs with edge selection probability $p_{\ell}(n)$ given in (A.23) and (A.24).

Clearly, $p_{\ell}(n), \ell=1,2$, meet the conditions in Theorem 2.8.6 [17, p. 67], which was stated earlier. Hence, the diameters of Erdös-Rényi random graphs $G\left(n, p_{\ell}(n)\right), n \in \mathbb{N}_{+}$, satisfy

$$
\frac{D\left(G\left(n, p_{\ell}(n)\right)\right)}{\log (n) / \log \left(n p_{\ell}(n)\right)} \stackrel{P}{\longrightarrow} 1, \ell=1,2,
$$

where $\stackrel{P}{\longrightarrow}$ denotes convergence in probability. Note that, for all $n \geq \tilde{n}(\epsilon)$, both $\zeta_{1}(n)$ and $\zeta_{2}(n)$ lie in the finite interval $\left[(\underline{\kappa}-\epsilon) e^{-\sqrt{2 /(\underline{\kappa}-\epsilon)}},(\bar{\kappa}+\epsilon) e^{\sqrt{2 /(\bar{\kappa}+\epsilon)}}\right]$. As a result, we have

$$
\frac{\log (n)}{\log \left(n p_{\ell}(n)\right)} \sim \frac{\log (n)}{\log (\log (n))}, \ell=1,2 .
$$

Equations (A.25) and (A.26) tell us

$$
\frac{D\left(G\left(n, p_{\ell}(n)\right)\right)}{\log (n) / \log (\log (n))} \stackrel{P}{\longrightarrow} 1, \ell=1,2 .
$$
we have

To complete the proof, we will prove that, with probability tending to one as $n \rightarrow \infty$,

$$
D\left(G\left(n, p_{2}(n)\right)\right) \leq D(\mathbb{G}(n, k(n))) \leq D\left(G\left(n, p_{1}(n)\right)\right) .
$$

To this end, we will show

$$
\lim _{n \rightarrow \infty} \mathbb{P}\left[\mathcal{E}_{E R, 1}^{(n)} \subseteq \mathcal{E}^{(n)} \subseteq \mathcal{E}_{E R, 2}^{(n)}\right]=1,
$$

which implies (A.28).

Define

$$
\hat{A}^{(n)}=\left\{\left(U_{i, k(n)}^{(n)}-\log \left(\frac{n-1}{k(n)}\right)\right) \in \hat{\Lambda}(n) \forall i \in \mathcal{V}^{(n)}\right\}
$$

where

$$
\hat{\Lambda}(n):=\left(-\sqrt{\frac{2}{\kappa(n)}}, \sqrt{\frac{2}{\kappa(n)}}\right) .
$$

Following similar steps as in the proof of Lemma A.1 (in Appendix B), we can show $\lim _{n \rightarrow \infty} \mathbb{P}\left[\hat{A}^{(n)}\right]=1$.

It is clear that, in the event $\hat{A}^{(n)}$, the edge set $\mathcal{E}^{(n)}$ of $\mathbb{G}(n, k(n))$ satisfies (A.29); conditional on the event $\hat{A}^{(n)}$, (a) all edges $(i, j)$ with $V_{i, j}>\log ((n-1) / k(n))+\sqrt{2 / \kappa(n)}$ belong to $\mathcal{E}^{(n)}$, and (b) no edge $(i, j)$ such that $V_{i, j} \leq \log ((n-1) / k(n))-\sqrt{2 / \kappa(n)}$ is in $\mathcal{E}^{(n)}$. 
The theorem is then a corollary of the following facts:

i. $\mathbb{P}\left[\hat{A}^{(n)}\right] \rightarrow 1$ as $n \rightarrow \infty$;

ii. In the event $\hat{A}^{(n)}$, inequalities in (A.28) hold; and

iii. The diameters $D\left(G\left(n, p_{\ell}(n)\right)\right), \ell=1,2$, satisfy (A.27).

\section{REFERENCES}

[1] R. Albert and A.-L. Barabási. "Statistical Mechanics of Complex Networks." Review of Modern Physics 74:1 (2002), 47-97.

[2] J. E. Angus. "The Probability Integral Transform and Related Results." SIAM Review 36:4 (1994), 652-654.

[3] M. J. B. Appel and R. P. Russo. "The Connectivity of a Graph on Uniform Points on [0, 1] $]^{d}$." Statistics \& Probability Letters 60 (2002), 351-357.

[4] J. Baillieul and P. J. Antsaklis. "Control and Communication Challenges in Networked RealTime Systems." Proceedings of the IEEE 95 (2007), 9-28.

[5] P. Balister, B. Bollobás, and A. Sarkar. "Percolation, Connectivity, Coverage and Colouring of Random Geometric Graphs.” In Handbook of Large-Scale Random Networks. Bolyai Society Mathematical Studies/Springer, 2008.

[6] P. Balister, B. Bollobás, A. Sarkar, and M. Walters. "Connectivity of Random $k$-NearestNeighbour Graphs." Advances in Applied Probability 37 (2005), 1-24.

[7] P. Balister, B. Bollobás, A. Sarkar, and M. Walters. "A Critical Constant for the $k$-NearestNeighbour Model." Advances in Applied Probability 41 (2009), 1-12.

[8] S. R. Blackburn and S. Gerke. "Connectivity of the Uniform Random Intersection Graph." Discrete Mathematics 309:16 (2009), 5130-5140.

[9] M. Boguñá and R. Pastor-Satorras. "Class of Correlated Random Networks with Hidden Variables." Physical Review E 68, article 036112, 2003.

[10] B. Bollobás. Random Graphs, (2nd ed.). Cambridge Studies in Advanced Mathematics, 73. Cambridge, UK: Cambridge University Press, 2001.

[11] M. R. Brito, E. L. Chávez, A. J. Quiroz, and J. E. Yukich. "Connectivity of the Mutual $k$ Nearest-Neighbor Graph in Clustering and Outlier Detection." Statistics \& Probability Letters 35 (1997), 33-42.

[12] G. Caldarelli, A. Capocci, P. De Los Rios and M.A. Muñoz. "Scale-Free Networks From Varying Vertex Intrinsic Fitness." Physical Review Letters 89:25 (2002), article 258702.

[13] T. Clausen and P. Jacquet. "Optimized Link State Routing Protocol (OLSR)." RFC 3626 Available online (http://www.rfc-base.org/rfc-3626.htm), 2003.

[14] H. A. David and H. N. Nagaraja. Order Statistics, (3rd ed.). New York: Wiley, 2003.

[15] L. de Haan and A. Ferreira. Extreme Value Theory: An Introduction. Springer Series in Operations Research and Financial Engineering, Springer. 2006.

[16] P. Diaconis and D. Freedman. "Finite Exchangeable Sequences." The Annals of Probability 8:4(1980),745-764, .

[17] R. Durrett. Random Graph Dynamics. Cambridge Series in Statistical and Probabilistic Mathematics. New York: Cambridge University Press, 2007.

[18] D. Easley and J. Kleinberg. Networks, Crowds, and Markets: Reasoning About a Highly Connected World. Cambridge, UK: Cambridge University Press, 2010.

[19] P. Erdös and A. Rényi. “On Random Graphs. I," Publicationes Mathematicae 6:(1959), 290297.

[20] P. Erdös and A. Rényi. "The Evolution of Random Graphs." Magyar Tud. Akad. Mat. Kutató Int. Közl. 5 (1960), 17-61.

[21] L. Eschenauer and V. D. Gligor. "A Key-Management Scheme for Distributed Sensor Network." In Proc. of the ACM Conference on Computer and Communications Security (CSS), pp. 41-47, Washington, DC: ACM, 2002. 
[22] M. Falk. "A Note on Uniform Asymptotic Normality of Intermediate Order Statistics." Annals of the Institute of Statistical Mathematics 41:1 (1989), 19-29.

[23] T. I. Fenner and A.M. Frieze. "On the Connectivity of Random $m$-Orientable Graphs and Digraphs." Combinatorica 2:4 (1982), 347359.

[24] E. N. Gilbert. "Random Graphs.” The Annals of Mathematical Statistics 30:4 (1959), 11411144.

[25] S. Goyal. Connections: An Introduction to the Economics of Networks. Princeton, NJ: Princeton University Press, 2009.

[26] G. Grimmett and D. Stirzaker. Probability and Random Processes (3rd ed.). New York: Oxford University Press, 2001.

[27] P. Gupta and P. R. Kumar. "Critical Power for Asymptotic Connectivity in Wireless Networks." In Stochastic Analysis, Control, and Optimization and Applications, pp. 547-566. Boston, MA: Birkhauser, 1998.

[28] W. P. M. H. Heemels, A. R. Teel, N. van de Wouw, and D. Nešić. "Networked Control Systems with Communication Constraints: Tradeoffs Between Transmission Intervals, Delays and Performance." IEEE Trans. on Automatic Control 55:8 (2010), 1781-1796.

[29] J. P. Hespanha, P. Naghshtabrizi and Y. Xu. "A Survey of Recent Results in Networked Control Systems." Proceedings of the IEEE 95:1 (2007), 138-162.

[30] Y. Isukapalli and B. D. Rao. "An Analytically Tractable Approximation for the Gaussian QFunction." IEEE Communications Letters 12:9 (2008), 668-671.

[31] M.O. Jackson. Social and Economic Networks. Princeton, NJ: Princeton University Press, 2010.

[32] S. Janson and M.J. Luczak. "A New Approach to the Giant Component Problem." Random Structures and Algorithms 34:2 (2009), 197-216.

[33] R. J. La. "Network Connectivity with Heterogeneous Mobility." Paper presented at Proc. of IEEE International Conference on Communications (ICC), Ottowa, Canada, Jun 2012.

[34] R. J. La and E. Seo. "Network connectivity with a Family of Group Mobility Models." IEEE Transactions on Mobile Computing 11:3 (2012), 504-517.

[35] A. M. Makowski and O. Yağan. "Scaling Laws for Connectivity in Random Threshold Graph Models with Non-Negative Fitness Variables." IEEE Journal on Selected Areas in Communications 31:9 (2013), 573-583.

[36] M. Molloy and B. Reed. "A Critical Point for Random Graphs with a Given Degree Sequence." Random Structures and Algorithms 6:2-3 (1995), 161-180.

[37] M. Molloy and B. Reed. "The Size of the Largest Component of a Random Graph on a Fixed Degree Sequence." Combinatorics, Probability and Computing 7:3 (1998), 295-305.

[38] M. Newman. Networks: An Introduction. New York: Oxford University Press, 2010.

[39] M. D. Penrose. Random Geometric Graphs. Oxford Studies in Probability, 5. Oxford, UK: Oxford University Press, 2003.

[40] C. Perkins and P. Bhagwat. "Highly Dynamic Destination-Sequenced Distance-Vector Routing (DSDV) for Mobile Computers." Computer Communications Review 24:4 (1994), 234-244.

[41] M. Shaked and J. G. Shanthikumar. Stochastic Orders, Springer Series in Statistics. New York: Springer-Verlag, 2007.

[42] F. Xue and P. R. Kumar. "The Number of Neighbors Needed for Connectivity of Wireless Networks." Wireless Networks 10: (2004), 169-181.

[43] O. Yağan and A.M. Makowski. "Zero-One Laws for Connectivity in Random Key Graphs." IEEE Transactions on Information Theory 58:5 (2012), 2983-2999. 